\title{
Concentration Flux Dependent on Radiative MHD Casson Flow with Arrhenius Activation Energy: Homotopy Analysis Method (HAM) with an Evolutionary Algorithm
}

\author{
Kohilavani Naganthran ${ }^{1}$, Ahmad Zeeshan², Md. Faisal Md. Basir ${ }^{3}$, Nasir Shehzad ${ }^{2}$, Roslinda Nazar ${ }^{1}$, Rakesh \\ Choudhary $^{4 *}$, Sankarasubramanian Balaji ${ }^{5}$ \\ ${ }^{1}$ Centre for Modelling and Data Science, Faculty of Science \& Technology, Universiti Kebangsaan Malaysia, UKM Bangi \\ 43600, Selangor, Malaysia \\ ${ }^{2}$ Department of Mathematics and Statistics, International Islamic University, Islamabad 44000, Pakistan \\ ${ }^{3}$ Department of Mathematical Sciences, Faculty of Science, Universiti Teknologi Malaysia, UTM Johor Bahru 81310, Johor, \\ Malaysia \\ ${ }^{4}$ School of General Education, Bhartiya Skill Development University, Jaipur 302037, India \\ ${ }^{5}$ Department of Mathematics, SASTRA Deemed University, Thanjavur 613401, India
}

Corresponding Author Email: rakesh.choudhary@ruj-bsdu.in

https://doi.org/10.18280/ijht.380403

Received: 22 February 2020

Accepted: 18 October 2020

\section{Keywords:}

Stefan blowing, blood flow, Casson fluid, HAM, Arrhenius activation energy

\begin{abstract}
Heat transfer analysis in nanofluids is an active research field due to its extraordinary physical and chemical properties. In the current study, the focus lies on the effects of Stefan blowing when a non-Newtonian Casson base fluid flows over a surface which stretches linearly. A uniform transverse magnetic field is employed. The chemical reaction in the fluid with activation energy and radiation effects have also been engaging the attention. Fundamental laws of conservation are employed to model governing equations of flow. Similarity transform is introduced to reduce the said system of partial differential equations to ordinary differential equations which are in turn tackled analytically using Homotopy Analysis Method with genetic algorithms to optimise the series solution. The impact of pertaining parameters on the dimensionless velocity, temperature and concentration were presented explicitly. This study relevant to remedies for malign tissues, cells or clogged arteries of the heart.
\end{abstract}

\section{INTRODUCTION}

Non-Newtonian fluids are the center of attention of many engineers and scientists due to their wide variety of applications in biomechanics, engineering, and industry like the extraction of crude oil from petroleum products. The flow of blood in the body and transport of sewage, etc. Working in the field of non-Newtonian fluid flows challenges the mathematician and simulation engineers with its diversity and complexity. As the complexity of such fluids offers no unique constitutive equation which encounters all the properties of such fluids, resulting in several non-Newtonian models are presented [1-5]. In non-Newtonian fluids, Casson fluid have several properties. Casson fluid illustrated yield stresses. Its mathematical modelling was derived by Casson in 1995. The model helps to improve the accountability of adhesive slurry at a high shear - rate. Casson fluid is a fluid that exerts shearthinning liquid which shows zero shear rate at infinite viscosity leads to no flow below particular yield stress (i.e., it behaves like a solid) [6]. Examples include tomato sauce, honey and jelly etc. Researchers had proven that blood rheology closest to Casson fluids [7].

Nadeem et al. [8] studied the three-dimensional flow of Casson fluid in porous space over a stretching sheet which is stretched linear under the effect of the uniform magnetic field. Tu and Deville [9] earlier show blood like the behaviour of Casson fluid and discussed pulsatile flow in arterial stenoses. More recently, Majeed et al. [10] investigated the influence of higher-order wall slip of Casson fluid along with heat transfer and suction/injection. They also consider the uniform magnetic field and convective thermal boundary condition. Animasaun et al. [11] examined the flow of Casson liquid over a paraboloid with the stratified melting surface. Some recent studies regarding Casson fluids are found in the papers [12$15]$.

Engineering application like paper drying, thermal coating, hot rolling, glass fiber stretching, etc. divert one's focus towards mass and heat transfer of non-newtonian fluids over a stretching sheet. Crane [16] seems to be the first to identify and investigate the boundary layer flow due to linear stretching. Motivated by this, it stimulates an extensive literature in this field, which includes, analysis over a stretching cylinder and sheet with Cattaneo-Christov heat flux model [17, 18]. The results of the stretching cylinder can be reduced to the sheet if the curvature of the cylinder is minimised. Heat transfer with multiple modes in the presence of electric and buoyancy effects can be found in the papers [19, 20]. Different nonNewtonian Rheological fluids include [21-23]. Magnetic rheological behaviour $[24,25]$ and different types of stretching can be found in the papers [26, 27].

Mehmood presented some new solutions and similarities for these flows [28]. An interesting aspect of mass and heat transfer of non-Newtonian fluids are a chemical reaction which arises in many industrial processes. The chemical reaction sorts an essential role in the manufacturing of instruments like a solar antenna, rubbery isolation, humidity in 
the gardens or dispersion of prescription in the blood. The first-order chemical reaction is proportional to the concentration of the fluid. Numerous readings linked to the present topic is available with diverse geometries [29-32].

However, given literature, not much is deliberated in the area of the mass transfer due to Arrhenius energy and binary chemical reaction. Bestman [33] was the first who examined the convective heat flow over a porous plate along with an activation energy. One of the essential components of a chemical reaction is its activation energy (below which the process is inert). The profound applications in geothermal reservoirs and oil industry make it an essential topic. Makinde et al. [34] worked with time-dependent flow through a porous space. Endo/exothermic reactions were explored by Maleque [35]. Awad et al. [36] employed SRM to find the solutions of rotating unsteady flow over an impulsively stretched sheet. For the stagnation point flow of Casson fluid, the work of Abbas et al. [37] is available. Mustafa et al. [38] discussed MHD nanofluid flow and Ellahi et al. [39] worked with Biofluid through flexible walls. In a process like paper dying via evaporation, sometimes occurs the effects of blowing, which are observed in Stefan's problem [40].

This is different from transpiration at the surface as it can be observed on the impermeable wall. It tends to generate the bulk motion of the mass, and consequently, other motion in a fluid is observed. Fang and Jing [41] have presented the effects on starching plate. Latiff et al. [42] work with rotating the solid disk. Alamri et al. [43] examined the effects in internal flow with slip effects. Uddin et al. [44] discuss Stefan's blowing on moving plate with nanofluid.

A review of the relevant literature indicates that there have been no previous studies which considered the Casson fluid flow over a radiative stretching sheet with binary chemical reaction with activation energy and the effect of heat source/sink and Stefan blowing. The solution of coupled nonlinear ODEs is handled by the homotopy method [45-47]. In the following sections, a mathematical model for the said problem is first developed, then in section three the analytical solution and its convergence is analysed, notable finds and results along with the comprehensive discussion is presented in section four, examined through graphs.

The main contributions of the paper are to:

-Expand the work of Maleque [35] to consider multiple convective and Stefan blowing boundary conditions.

-Utilize HAM with a genetic algorithm to arrive at the solutions.

-Scrutinise the impacts of different parameters on the velocity, temperature and concentration profiles of Casson fluids through graphs.

-Obtain an accurate solution from the proposed method could be a stepping stone to establishing mathematical formulations to describe the behaviour of the heat transfer and mass transport process.

\section{MATHEMATICAL FRAME}

The stagnation point flow of non-Newtonian Casson fluid with nanoparticles through a uniform magnetic field endure binary chemical reaction over a horizontal sheet stretched linearly is the matter of interest here. The essential component of a chemical reaction is the activation energy. Hence, Arrhenius activation energy, along with radiation, is also incorporated. It is assumed that fluid saturates the region above the sheet $(y>0)$, where the sheet is placed along the $x$ axis $(\mathrm{y}=0)$ and a uniform magnetic field is an applied parallel to the $y$-axis (see Figure 1). Further assumptions are that fluid conducts electricity and induced magnetic field is taken to be negligible as magnetic Reynolds number is small.

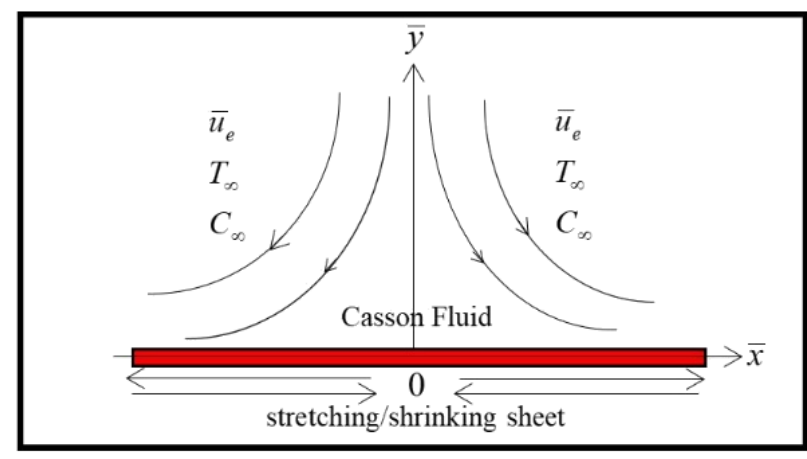

Figure 1. A schematic diagram of the problem (Adapted from Shehzad et al. [32])

Now, considering the temperature on the surface of the sheet and far away is $T_{f}$ and $T_{\infty}$ respectively. Similarly, concentrations are $C_{f}$ and $C_{\infty}$ respectively. The mixed convective radiative heat flow is observed along with heat source/sink. On the stretching surface, Stefan blowing effects are also included. The rheological constitutive equation for Casson fluid is [37].

$$
\tau_{i j}= \begin{cases}2\left(\frac{P_{y}}{\sqrt{2 \pi}}+\mu_{B}\right), & \pi>\pi_{c} \\ 2\left(\frac{P_{y}}{\sqrt{2 \pi_{c}}}+\mu_{B}\right), & \pi<\pi_{c}\end{cases}
$$

$\pi=e_{i j}, e_{i j}$ is the $(i, j)$ th component of the deformation rate. The governing flow equations in the component form are [32]:

$$
\begin{gathered}
\frac{\partial v}{\partial y}+\frac{\partial u}{\partial x}=0 \\
v\left(1+\frac{1}{\beta}\right) \frac{\partial^{2} u}{\partial y^{2}}+u_{e} \frac{d u_{e}}{d x}+\frac{\sigma B_{0}^{2}\left(u_{e}-u\right)}{\rho}=u \frac{\partial u}{\partial x}+v \frac{\partial u}{\partial y} \\
\alpha \frac{\partial^{2} T}{\partial y^{2}}+\frac{Q_{0}\left(T-T_{\infty}\right)}{\left(\rho c_{p}\right)_{f}}+\frac{16 \sigma_{1} T_{\infty}^{3}}{3 k^{*}\left(\rho c_{p}\right)_{f}} \frac{\partial^{2} T}{\partial y^{2}}=u \frac{\partial T}{\partial x}+v \frac{\partial T}{\partial y} \\
D \frac{\partial^{2} C}{\partial y^{2}}-k_{r}^{2}\left(C-C_{\infty}\right)\left(\frac{T}{T_{\infty}}\right)^{m} \exp \left(\frac{-E_{a}}{K^{*} T}\right)=u \frac{\partial C}{\partial x}+v \frac{\partial C}{\partial y}
\end{gathered}
$$

The conditions at the boundary are given as $[10,43]$ :

$$
\begin{gathered}
u=u_{w}=b x, v=-\frac{D}{\left(1-C_{f}\right)} \frac{\partial C}{\partial y}, \\
-k_{1} \frac{\partial T}{\partial y}=\left(-h_{1} T+h_{1} T_{f}\right), \\
-k_{2} \frac{\partial C}{\partial y}=\left(-h_{2} C+h_{2} C_{f}\right) \text { at } y=0 \\
u=u_{e}=c x, T=T_{\infty}, C=C_{\infty} \text { as } y \rightarrow \infty
\end{gathered}
$$


Considering the following the similarity variables [37]:

$$
\begin{gathered}
\eta=\sqrt{\frac{c}{v}} y, u=c x f^{\prime}(\eta), v=-\sqrt{c v} f(\eta), \\
\theta(\eta)=\frac{T-T_{\infty}}{T_{f}-T_{\infty}}, \phi(\eta)=\frac{C-C_{\infty}}{C_{f}-C_{\infty}} .
\end{gathered}
$$

Making use of (8), governing Eqns. (3) to (5) converted into the system of ordinary differential equations, obtained:

$$
\begin{gathered}
M\left(1-f^{\prime}\right)+1+f f^{\prime \prime}-f^{\prime 2}+\left(1+\frac{1}{\beta}\right) f^{\prime \prime \prime}=0 \\
\frac{(1+R)}{\operatorname{Pr}} \theta^{\prime \prime}+f \theta^{\prime}+Q_{c} \theta=0 \\
\frac{1}{S c} \phi^{\prime \prime}+f \phi^{\prime}-K_{E}\left(1+\theta \theta_{w}\right)^{m} \phi \exp \left(\frac{-E}{1+\theta \theta_{w}}\right)=0
\end{gathered}
$$

The transformed conditions at the boundary are as follows

$$
\begin{gathered}
f^{\prime}(0)=\lambda, f(0)=s_{1} \frac{1}{S c} \phi^{\prime}, \theta^{\prime}(0)=-B i_{1}(1-\theta(0)), \\
\phi^{\prime}(0)=-B i_{2}(1-\phi(0)), \\
f^{\prime}(\infty)=1, \theta(\infty)=0, \phi(\infty)=0
\end{gathered}
$$

Here the $s_{1}>0$ presents mass blowing at the sheet wall and $s_{1}<0$, presents the mass suction. Also, we fixed $\operatorname{Pr}=21[48,49]$, which refers to human blood. All the parameters are involved are used explained in the nomenclature section. The physical quantities of interest in given as follows: (Shehzad et al. [32])

$$
\begin{gathered}
C_{f}=\left.\frac{1}{\rho u_{w}^{2}}\left(\mu_{B}+\frac{P_{y}}{\sqrt{2 \pi}}\right) \frac{\partial u}{\partial y}\right|_{y=0} \\
N u_{x}=\frac{q_{w} x}{k\left(T_{f}-T_{\infty}\right)} \\
S h_{x}=\frac{J_{w} x}{D_{B}\left(C_{f}-C_{\infty}\right)},
\end{gathered}
$$

where, $q_{w}=-\left.\left(k+\frac{16 \sigma_{1} T_{\infty}^{3}}{3 k^{*}\left(\rho c_{p}\right)_{f}}\right) \frac{\partial T}{\partial y}\right|_{y=0}$ and $J_{w}=-\left.D_{B} \frac{\partial C}{\partial y}\right|_{y=0}$ are heat and mass flux, respectively. In the dimensionless form (14) - (16) can be presented as follows

$$
\begin{gathered}
f^{\prime \prime}(0)\left(1+\frac{1}{\beta}\right)=C_{f} \operatorname{Re}_{x}^{1 / 2} \\
-(1+R) \theta^{\prime}(0)=N u_{x} \operatorname{Re}_{x}^{-1 / 2} \\
-\phi^{\prime}(0)=S h_{x} \operatorname{Re}_{x}^{-1 / 2}
\end{gathered}
$$

where, $R e_{x}=\frac{u_{e} x}{v}$ signifies local Reynold number.

\subsection{Numerical procedure and its optimisation}

Considering Eqns. (9)-(11) and solving using HAM [49] the nth order deformation takes the form:

$$
\left.\begin{array}{l}
(1-q) L_{1}\left[f(\eta, \mathrm{q})-f_{0}(\eta)\right]=q \hbar_{1} N_{1}[f(\eta, q), \theta(\eta, q), \phi(\eta, q)], \\
(1-q) L_{2}\left[\theta(\eta, \mathrm{q})-\theta_{0}(\eta)\right]=q \hbar_{2} N_{2}[f(\eta, q), \theta(\eta, q), \phi(\eta, q)], \\
(1-q) L_{2}\left[\phi(\eta, \mathrm{q})-\phi_{0}(\eta)\right]=q \hbar_{3} N_{3}[f(\eta, q), \theta(\eta, q), \phi(\eta, q)] .
\end{array}\right\}
$$

To get an analytical solution, HAM is utilised Initial approximations $u_{0}(\eta), \theta_{0}(\eta), \phi_{0}(\eta)$ such that it satisfies boundary condition. The choice of initial guess is

$$
\left.\begin{array}{c}
f_{0}(\eta)=\frac{s_{1} B i_{2}}{S c\left(1+B i_{2}\right)}-(1-\lambda)+x+(1-\lambda) \operatorname{Exp}[-\eta], \\
\theta_{0}(\eta)=\frac{B i_{1}}{1+B i_{1}} \operatorname{Exp}[-\eta], \\
\phi_{0}(\eta)=\frac{B i_{2}}{1+B i_{2}} \operatorname{Exp}[-\eta],
\end{array}\right\}
$$

and the supplementary linear operators $L_{1}$ for velocity and $L_{2}$ for temperature and concentration profiles, which are

$$
\left.\begin{array}{l}
L_{1}=\frac{\partial^{3}}{\partial \eta^{3}}+\frac{\partial^{2}}{\partial \eta^{2}}, \\
L_{2}=\frac{\partial^{2}}{\partial \eta^{2}}-1,
\end{array}\right\}
$$

The corresponding solutions for the homogenous problem should be of the form.

$$
\left.\begin{array}{l}
f=\mathrm{c}_{1}+\mathrm{c}_{2} \eta+c_{3} \mathrm{e}^{-\eta}, \\
\theta=c_{4} \mathrm{e}^{-\eta}+c_{5} \mathrm{e}^{\eta}, \\
\phi=c_{6} \mathrm{e}^{-\eta}+c_{7} \mathrm{e}^{\eta}
\end{array}\right\}
$$
(13).

At each iteration, constant will be evaluated using Eq. (12)Finally, the nonlinear operators $N_{1}, N_{2}$ and $N_{3}$ for velocity, temperature and concentration wrote according to Eq. (9) through to (11) are:

$$
\begin{aligned}
& N_{1}[f(y, q), \theta(y, q), \phi(y, q)]=\left(1+\frac{1}{\beta}\right) f^{\prime \prime \prime}(y, q) \\
& -M\left(f^{\prime}(y, q)\right)-f^{\prime}(y, q)^{2}+f(y, q) f^{\prime \prime}(y, q)+\chi(1+M), \\
& N_{2}[f(y, q), \theta(y, q), \phi(y, q)]=\frac{(1+R)}{\operatorname{Pr}} \theta^{\prime \prime}(y, q) \\
& +f(y, q) \theta^{\prime}(y, q)+Q_{c} \theta(y, q), \\
& N_{3}[f(y, q), \theta(y, q), \phi(y, q)]=\frac{1}{S c} \phi^{\prime \prime}(y, q) \\
& +f(y, q) \phi^{\prime}(y, q)+K_{E} E\left(1+\theta(y, q) \theta_{w}\right)^{m-1} \phi(y, q) .
\end{aligned}
$$

Defining the following expansion

$$
\begin{aligned}
& f(y, q)=f_{0}(\mathrm{y})+\sum_{k=1}^{n} q^{k} f_{k}(y) \\
& \theta(y, q)=\theta_{0}(\mathrm{y})+\sum_{k=1}^{n} q^{k} \theta_{k}(y) \\
& \phi(y, q)=\phi_{0}(\mathrm{y})+\sum_{k=1}^{n} q^{k} \phi_{k}(y)
\end{aligned}
$$


Utilising the expensing arrangement characterised in term of $(f(y, q), \theta(y, q)$ and $\phi(y, q))$ as referenced in Eq. (25) into the Eq. (20). We get an arrangement of direct differential equations with their significant limit conditions. The solution deforms from initial guesses to approximate value when $q$ changes continuously from 0 to 1 . Here $\hbar_{1}, \hbar_{2}$ and $\hbar_{3}$ are convergence control parameters whose values are optimised to get the minimum "Residual Error" using a genetic algorithm. A flow chart is drawn below, showing the procedure followed in the optimisation process.

\subsection{Genetic algorithm}

The theory of inheritance and evolution of living organisms explains the evolution and survival of the fittest organism, not the strongest. Based on this theory, an optimisation technique evolves knows as "Genetic algorithm (GA)". It assumes some approximate values for the requested parameter (in this case convergence control parameter). These values called chromosomes first combined (crossover) to generate new daughter chromosomes. After the crossover process, some of its values are changed to initiate mutation. The so formed daughter chromosomes and parent chromosomes are tested for best fitness values. The process will be repeated until an optimum value is achieved (See Figure 2). Optimisation Tool GUI in Matlab is employed to find the convergence control parameters of HAM. The flow chart is drawn to explain the current method.

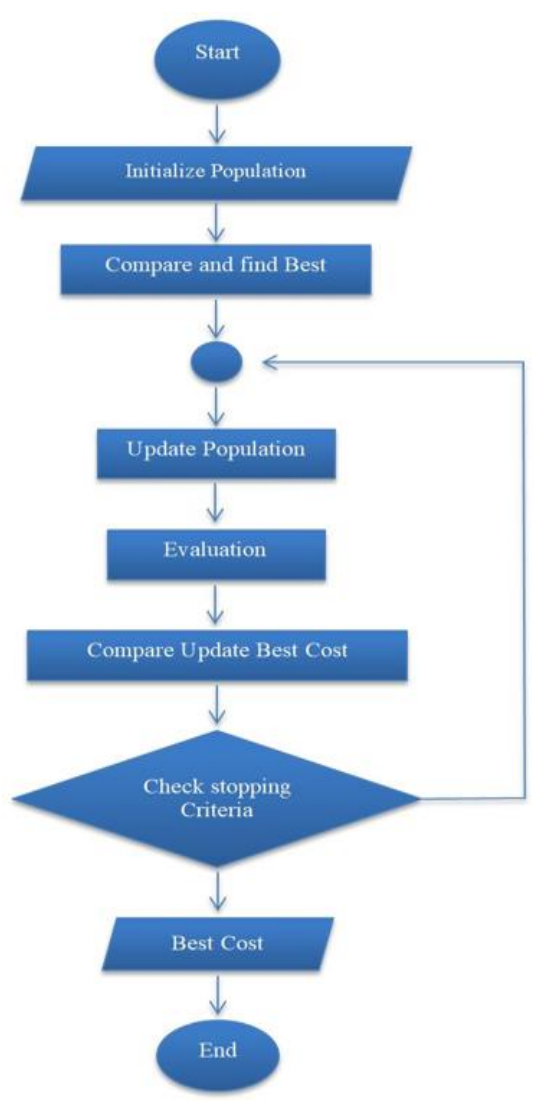

Figure 2. Flow chart of genetic algorithm

\section{RESULT AND DISCUSSION}

In this section, we investigated the behaviour of Casson fluid parameter $\beta$, chemical reaction parameter $K_{E}$, constant of temperature difference $\theta_{w}$, Stefan's blowing coefficient $s_{1}$ and activation energy $E$ on $f^{\prime}(\eta), \theta(\eta)$ and $\phi(\eta)$. Also, $C_{f} R e_{x}^{1 / 2}$ (skin friction), $N u_{x} R e_{x}^{-1 / 2}$ (Nusselt Number), $S h_{x} R e_{x}^{-1 / 2}$ (Sherwood number) are plotted to emulate the variation in the flow. The following discussion and results are obtained by using appropriate values of emerging parameters: $M=S c=0.2, B i_{1}, B i_{2}, Q_{c}=0.2, R=0.2, s_{1}, \lambda=1, S_{c}=0.7, \theta_{w}=7, E=1$, $K_{E}=1$ and $\beta=1$.

With the rise of $\beta$ the overall viscosity decline and as $\beta$ approaches infinity, the non-Newtonian behaviour tends to disappear, which lead to enhancement of the flow velocity as $\beta$ increases. Similar to the temperature profiles, with the increase of $\beta$ (Figure 3 ) the However, concentration decrease due to a reduction in viscosity and consequently, the reduction is resistances. Figure 4 shows variation observed in $f^{\prime}(\eta), \theta(\eta)$ and $\phi(\eta)$ when a slight variation in chemical reaction parameter $K_{E}$ is observed.

It is detected that the velocity of the fluid rises with growth in values of $K_{E}$. It is also realized that for generative reaction, i.e., $K_{E}<0$ fluid flow enhances. Whereas, in destructive reaction $K_{E}>0$, drop in the fluid flow is observed. Moreover, the temperature of nanofluid and volume fraction concentration tends to decrease as $K_{E}$ shows an increasing trend, which is due to the weak effect of buoyancy force concentration gradient. Figure 5 elucidates the increasing behaviour in the concentration of nanofluid when activation energy $E$ is increased.

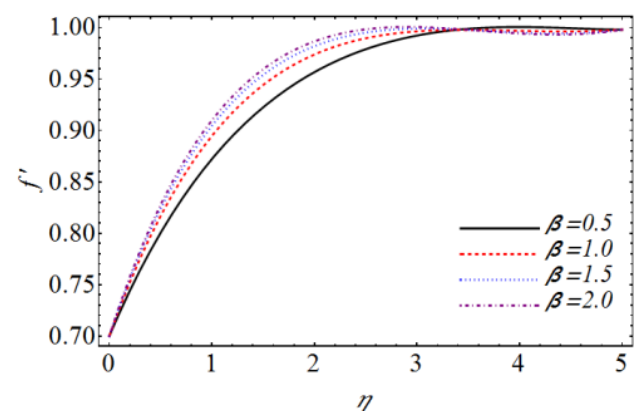

(a)

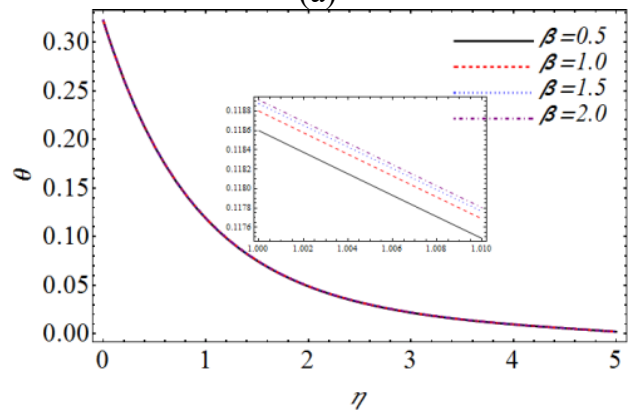

(b)

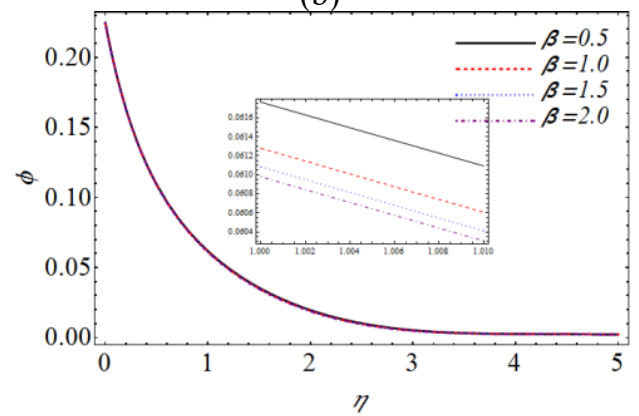

(c)

Figure 3. Effect of the Casson fluid parameter on a) velocity b) temperature c) concentration 


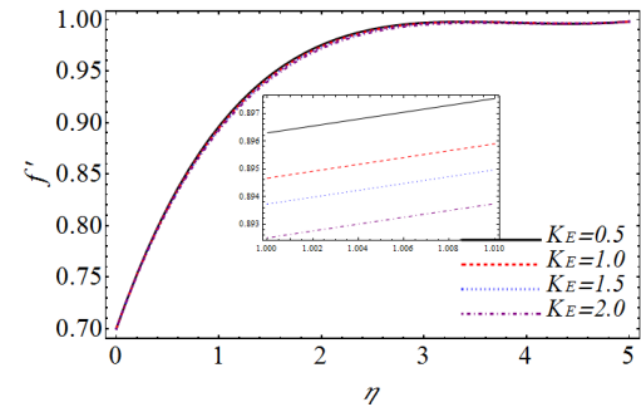

(a)

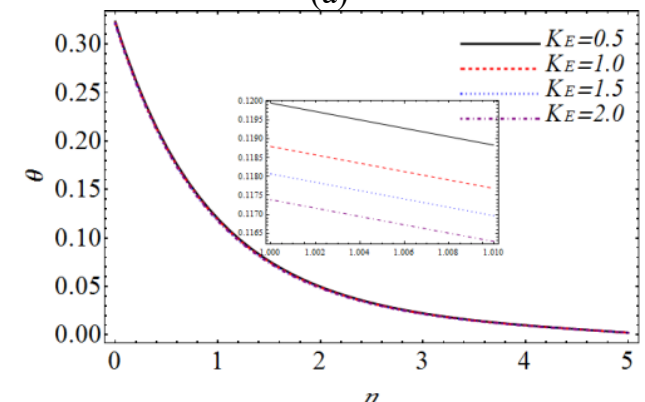

(b)

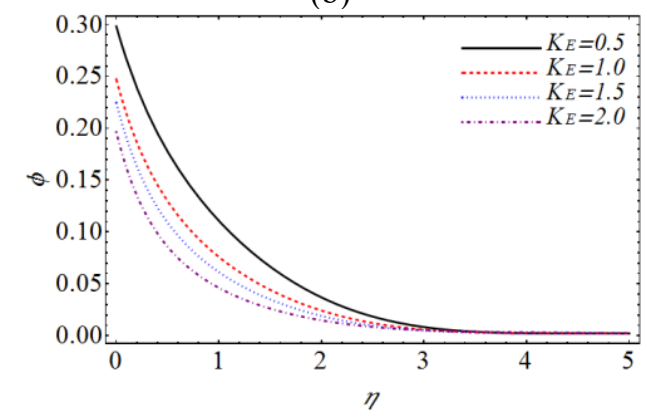

(c)

Figure 4. Effect of the chemical reaction on a) velocity b) temperature c) concentration

Physically, due to the higher activation energy, it takes the greater temperature to start a chemical reaction hence at a lower temperature, the reaction rate is less, consequently, reduce the chemical reaction. Increasing value $E$ also tends to reduce velocity and temperature.

Figure 6 demonstrate the impact of Stefan mass blowing or suction coefficient $s_{1}$. Here, it is found that velocity for larger values of $s_{1}$ reduces far away from the wall, while its boosts up in the neighborhood of walls as mass flow boosted the velocity. For temperature profile, it is apparent that the thermal performance reduces as the system is cooled in case of suction, so boundary layer thickness shrinkages. It is consequently blowing effects by raising the temperature. For concentration profile, Figure reflects that concentration function increased with the increase of $s_{1}$. The effects of $\beta$ and Stefan blowing parameter $s_{1}$ on $C_{f} R e_{x}^{1 / 2}$ are shown in Figure 7.

It is noted from said figure that the Stefan parameter and Casson fluid parameters have the same proportional to each other but opposite trend measure for skin friction. In results as the Casson and Stefan parameter increased, then the skin friction on the surface of geometry decreases. Figure 8 depicts the influences of temperature difference parameter $\theta_{w}$ and activation energy $E$ on $C_{f} R e_{x}^{1 / 2}$

$C_{f} R e_{x}^{1 / 2}$ is increasing with the increase of activation energy parameter but reverse behaviour for the temperature difference parameter is noted for skin friction. In Figures 9 and
10 show the effects of Casson fluid, Stefan blowing, temperature difference and activation energy parameters on Nusselt number.

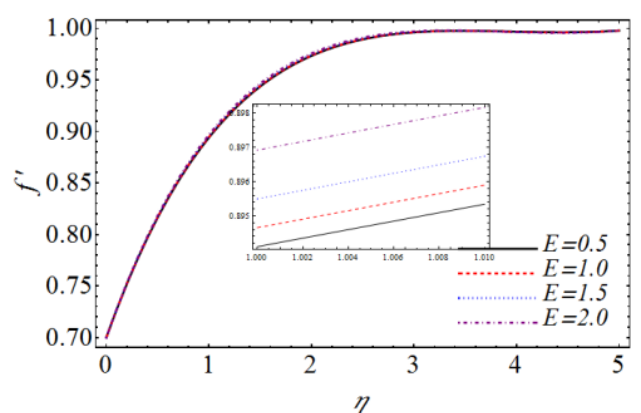

(a)

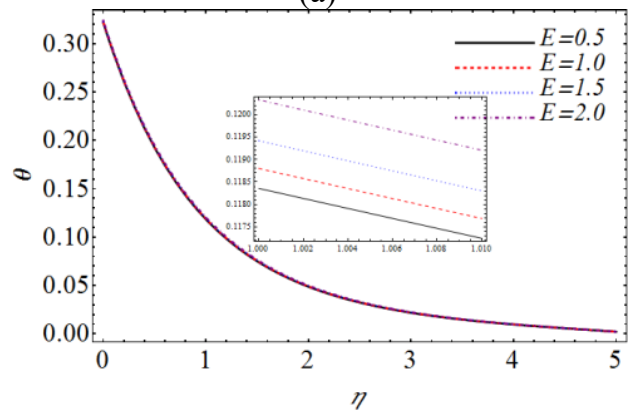

(b)

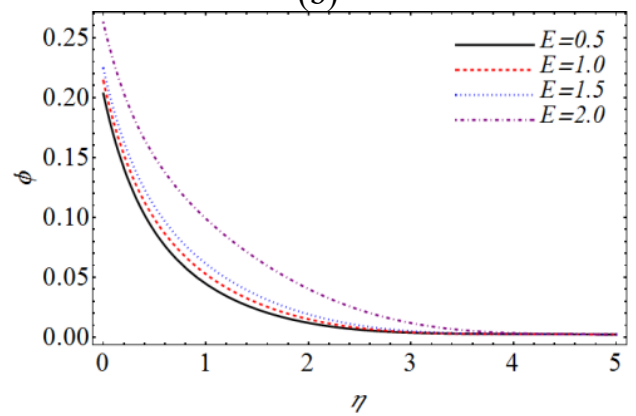

(c)

Figure 5. Effect of the activation energy on a) velocity b) temperature c) concentration

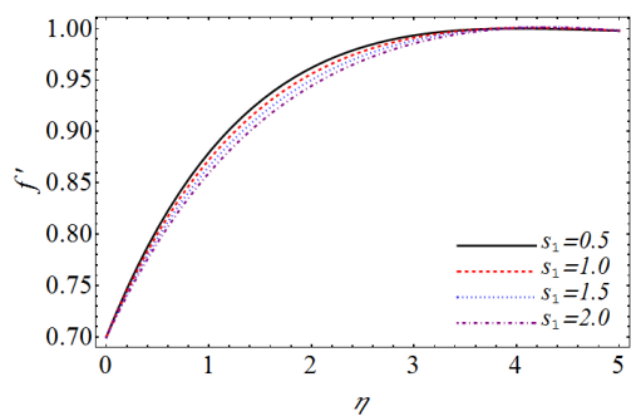

(a)

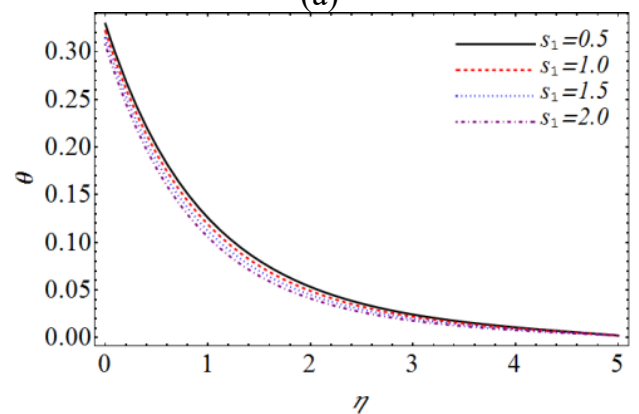

(b) 


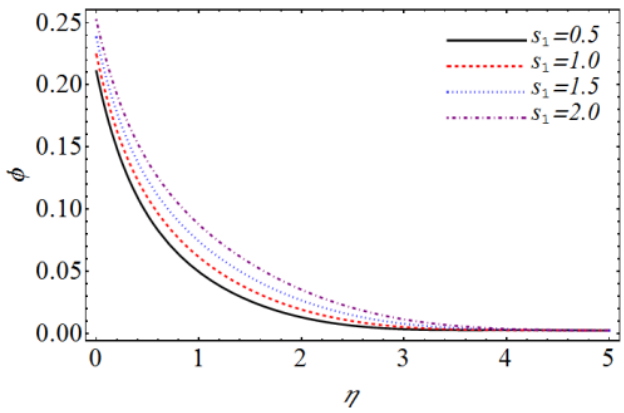

(c)

Figure 6. Effect of the Stefan mass blowing on a) velocity b) temperature c) concentration

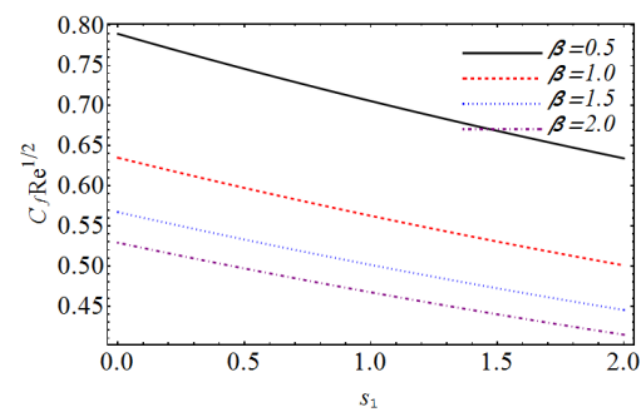

Figure 7. Effect of Casson fluid and Stefan parameters on the skin friction coefficient

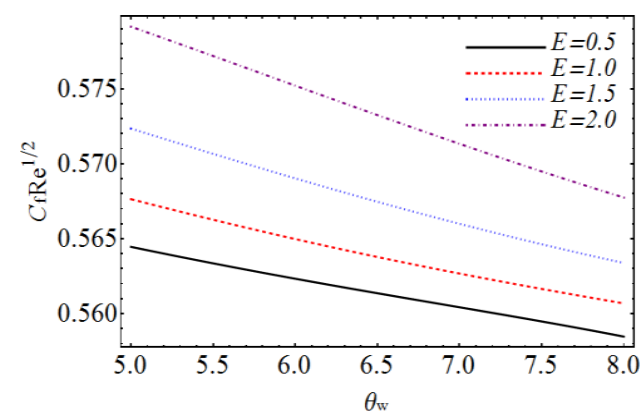

Figure 8. Effect of temperature difference and Activation Energy on the skin friction coefficient

In both Figures $9-10, N u_{x} R e_{x}^{-1 / 2}$ is growing up with the large values of Stefan blowing and temperature difference parameters but the drop in heat transfer is observed for large values of Casson fluid and activation energy parameters. Figure 11 scrutinizes the $S h_{x} R e_{x}^{-1 / 2}$ versus Stefan blowing parameter $s_{1}$ for different values of Casson fluid parameter $\beta$.

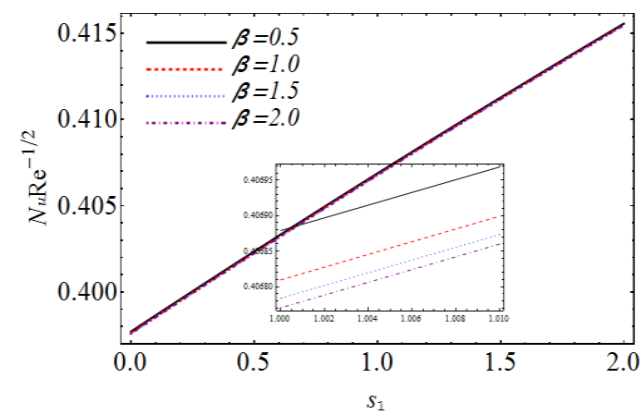

Figure 9. Effect of Casson fluid and Stefan parameters on the Nusselt number

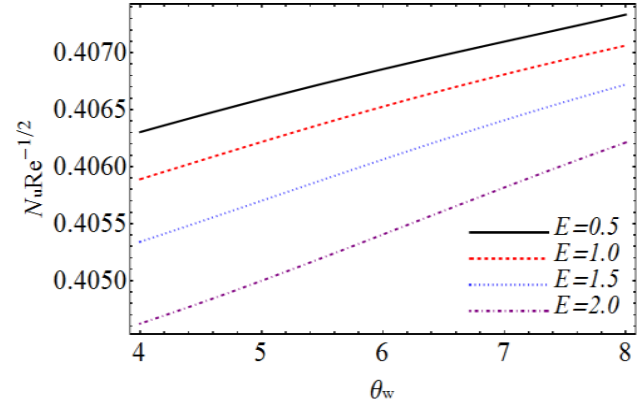

Figure 10. Effect of temperature difference and Activation Energy on the Nusselt number

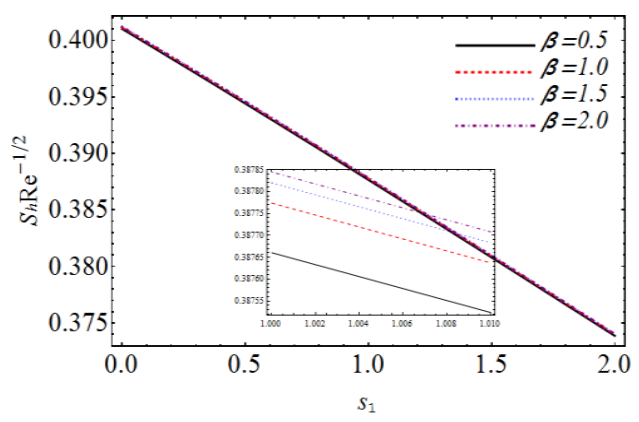

Figure 11. Effect of Casson fluid and Stefan parameters on the Sherwood number

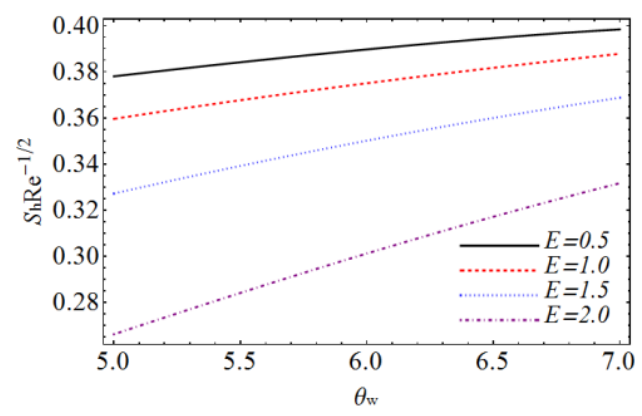

Figure 12. Effect of temperature difference and Activation Energy on the Sherwood number

The $S h_{x} R e_{x}^{-1 / 2}$ expressively rises with growth in Casson fluid parameter $\beta$. However, there is an exponential decay in $S h_{x} R e_{x}^{-1 / 2}$ when Stefan blowing is amplified. Figure 12 shows the effect of temperature difference parameter $\theta_{w}$ on the $S h_{x} R e_{x}^{-1 / 2}$ for variation of activation energy $E$. This figure exposes that wall mass flux has a reverse trend with $\theta_{w}$ and $E$.

\section{CONCLUSIONS}

The following conclusions can be drawn from the present exploration:

- Velocity decays for larger chemical reaction and Stefan blowing parameter while the reverse trend is noticed for the higher Casson fluid parameter, and activation energy.

- Concentration decreases as the Casson fluid parameter and chemical reaction are increased while the opposite behaviour is perceived for higher Stefan blowing and activation energy. 
- Temperature profile increases for larger Casson fluid parameter and activation energy, but the contradictory trend is observed for higher chemical reaction and Stefan blowing parameter.

- As in Table 1, surface drag force reduces for higher Casson fluid parameter and activation energy while the reverse behaviour is observed for higher temperature difference and Stefan blowing parameter. The reduced Nusselt number is directly proportional to the activation energy and inversely proportional to the temperature difference, Casson fluid parameter and Stefan blowing parameter. The reduced Sherwood number is inversely proportional to the temperature difference parameter, and an align increment to the activation energy, Casson fluid parameter and Stefan blowing parameter.

- As in Figures 7-12, the reduced Nusselt number towards temperature difference, Stefan blowing parameter are the highest, while the Casson fluid parameter and activation energy are at the minimal. Also, the reduced Sherwood number decreases toward the Stefan blowing and activation energy while reverse behaviour is noticed for higher temperature difference and Casson fluid parameter. The surface drag force is directly proportional to the activation energy and inversely proportional to the temperature difference, Casson fluid parameter and Stefan blowing parameter.

Table 1. Values $\left(1+\frac{1}{\beta}\right) f^{\prime \prime}(0),-(1+R) \theta^{\prime}(0),-\phi^{\prime}(0)$ as the governing parameters vary

\begin{tabular}{|c|c|c|c|c|c|c|}
\hline $\begin{array}{l}\text { Activation } \\
\text { energy, } E\end{array}$ & $\begin{array}{l}\text { Temperature } \\
\text { difference, } \theta_{w}\end{array}$ & $\begin{array}{c}\text { Casson } \\
\text { parameter, } \beta\end{array}$ & $\begin{array}{c}\text { Stefen } \\
\text { blowing, } s_{1}\end{array}$ & $\left(1+\frac{1}{\beta}\right) f^{\prime \prime}(0)$ & $-(\mathbf{1}+\boldsymbol{R}) \boldsymbol{\theta}^{\prime}(\mathbf{0})$ & $-\boldsymbol{\phi}^{\prime}(\mathbf{0})$ \\
\hline 0.5 & 0.5 & 1 & 0.75 & 0.576297 & 0.675464 & 0.298973 \\
\hline 0.75 & & & & 0.575685 & 0.67557 & 0.303952 \\
\hline \multirow[t]{7}{*}{1} & & & & 0.57505 & 0.675681 & 0.308992 \\
\hline & 0.75 & & & 0.576965 & 0.67535 & 0.29305 \\
\hline & 1 & & & 0.579098 & 0.674986 & 0.273698 \\
\hline & & 5 & & 0.411362 & 0.674156 & 0.387892 \\
\hline & & 10 & & 0.3855 & 0.674142 & 0.391307 \\
\hline & & & 0.5 & 0.397798 & 0.670338 & 0.394673 \\
\hline & & & 0.0 & 0.423833 & 0.662577 & 0.401282 \\
\hline
\end{tabular}

\section{ACKNOWLEDGMENT}

The authors from the Universiti Kebangsaan Malaysia would like to acknowledge the research university grant from the Universiti Kebangsaan Malaysia (project code: GUP2019-034).

\section{REFERENCES}

[1] Bird, R.B. (1976). Useful non-Newtonian models. Annual Review of Fluid Mechanics, 8(1): 13-34. http://dx.doi.org/10.1146/annurev.fl.08.010176.000305

[2] Ellahi, R., Zeeshan, A., Hussain, F., Abbas, T. (2019). Thermally charged MHD bi-phase flow coatings with non-Newtonian nanofluid and Hafnium particles along slippery walls. Coatings, 9(5): 300. http://dx.doi.org/10.3390/coatings9050300

[3] Hassan, M., Ellahi, R., Zeeshan, A., Bhatti, M.M. (2019). Analysis of natural convective flow of non-Newtonian fluid under the effects of nanoparticles of different materials. Proceedings of the Institution of Mechanical Engineers, Part E: Journal of Process Mechanical Engineering, 233(3): 643-652. http://dx.doi.org/10.1177/0954408918787122

[4] Hayat, T., Hussain, Z., Farooq, M., Alsaedi, A. (2018). Magnetohydrodynamic flow of Powell-Eyring fluid by a stretching cylinder with Newtonian heating. Thermal Science, 22: 371-82. http://dx.doi.org/10.2298/TSCI150717162H

[5] Reddy, J.R., Kumar, K.A., Sugunamma, V., Sandeep, N. (2018). Effect of cross diffusion on MHD nonNewtonian fluids flow past a stretching sheet with nonuniform heat source/sink: A comparative study.
Alexandria Engineering Journal, 57(3): 1829-38. http://dx.doi.org/10.1016/j.aej.2017.03.008

[6] Dash, R., Mehta, K., Jayaraman, G. (1996). Casson fluid flow in a pipe filled with a homogeneous porous medium. International Journal of Engineering Science, 34(1): 1145-56. http://dx.doi.org/10.1016/00207225(96)00012-2

[7] Fung, Y. (1984). Biodynamics: Circulation SpringerVerlag. New York. https://doi.org/10.1007/978-1-47573884-1

[8] Nadeem, S., Haq, R.U., Akbar, N.S., Khan, Z.H. (2013). MHD three-dimensional Casson fluid flow past a porous linearly stretching sheet. Alexandria Engineering Journal, 52(4):

577-82. http://dx.doi.org/10.1016/j.aej.2013.08.005

[9] Tu, C., Deville, M. (1996). Pulsatile flow of nonNewtonian fluids through arterial stenoses. Journal of biomechanics, 29(7): http://dx.doi.org/10.1016/0021-9290(95)00151-4

[10] Majeed, A., Zeeshan, A., Mahmood, T., Rahman, S.U., Khan, I. (2019). Impact of magnetic field and secondorder slip flow of Casson liquid with heat transfer subject to suction/injection and convective boundary condition. Journal of Magnetics, 24(1): 81-89. http://dx.doi.org/10.4283/JMAG.2019.24.1.081

[11] Animasaun, I., Makinde, O., Saleem, S. (2019). Mixed convection flow of Newtonian fluids over an upper horizontal thermally stratified melting surface of a paraboloid of revolution. Journal of the Brazilian Society of Mechanical Sciences and Engineering, 41(4): 197. http://dx.doi.org/10.1007/s40430-019-1698-7

[12] Ijaz, N., Ahmed, Z., Rehman, S. (2018). Effect of electroosmosis and mixed convection on nano-bio-fluid with non-spherical particles in a curved channel. Mechanics \& 
Industry,

19(1):

108.

http://dx.doi.org/10.1051/meca/2017040

[13] Raju, C.S., Sandeep, N., Ali, M.E., Nuhait, A.O. (2019). Heat and mass transfer in 3-D MHD Williamson-Casson fluids flow over a stretching surface with non-uniform heat source/sink. Thermal Science, 23(1): 281-293. http://dx.doi.org/10.2298/TSCI160426107R

[14] Rehman, K.U., Malik, M., Khan, W.A., Khan, I., Alharbi, S. (2019). Numerical solution of non-Newtonian fluid flow due to rotatory rigid disk. Symmetry, 11(5): 699. http://dx.doi.org/10.3390/sym11050699

[15] Vijayalakshmi, P., Gunakala, S.R., Animasaun, I., Sivaraj, R. (2019). Chemical reaction and nonuniform heat source/sink effects on Casson fluid flow over a vertical cone and flat plate saturated with porous medium. Applied Mathematics and Scientific Computing: Springer, 117-27. http://dx.doi.org/10.1007/978-3-03001123-9_13

[16] Crane, L.J. (1970). Flow past a stretching plate. Zeitschrift für angewandte Mathematik und Physik ZAMP, 21(4):

645-7.

http://dx.doi.org/10.1007/BF01587695

[17] Alamri, S.Z., Khan, A.A., Azeez, M., Ellahi, R. (2019). Effects of mass transfer on MHD second grade fluid towards stretching cylinder: A novel perspective of Cattaneo-Christov heat flux model. Physics Letters A, 383(2-3):

276-81. http://dx.doi.org/10.1016/j.physleta.2018.10.035

[18] Sui, J., Zheng, L., Zhang, X. (2016). Boundary layer heat and mass transfer with Cattaneo-Christov doublediffusion in upper-convected Maxwell nanofluid past a stretching sheet with slip velocity. International Journal of Thermal Sciences, 104: 461-8. http://dx.doi.org/10.1016/j.ijthermalsci.2016.02.007

[19] Aybar, H.Ş., Sharifpur, M., Azizian, M.R., Mehrabi, M., Meyer, J.P. (2015). A review of thermal conductivity models for nanofluids. Heat Transfer Engineering, 36(13): http://dx.doi.org/10.1080/01457632.2015.987586

[20] Zeeshan, A., Ismael, H., Yousif, M., Mahmood, T., Rahman, S. (2018). Simultaneous effects of slip and wall stretching/shrinking on radiative flow of magneto nanofluid through porous medium. Journal of Magnetics, 23(4):

491-8. http://dx.doi.org/10.4283/JMAG.2018.23.4.491

[21] Gireesha, B., Gorla, R.S.R., Mahanthesh, B. (2015) Effect of suspended nanoparticles on three-dimensional MHD flow, heat and mass transfer of radiating EyringPowell fluid over a stretching sheet. Journal of Nanofluids, 4(4): 474-484. http://dx.doi.org/10.1166/jon.2015.1177

[22] Narayana, P.S., Babu, D.H. (2016). Numerical study of MHD heat and mass transfer of a Jeffrey fluid over a stretching sheet with chemical reaction and thermal radiation. Journal of the Taiwan Institute of Chemical Engineers, 59: 18-25. http://dx.doi.org/10.1016/j.jtice.2015.07.014

[23] Sheikholeslami, M., Rokni, H.B. (2018). CVFEM for effect of Lorentz forces on nanofluid flow in a porous complex shaped enclosure by means of non-equilibrium model. Journal of Molecular Liquids, 254: 446-642. http://dx.doi.org/10.1016/j.molliq.2018.01.130

[24] Hassan, M., Fetecau, C., Majeed, A., Zeeshan, A. (2018). Effects of iron nanoparticles' shape on convective flow of ferrofluid under highly oscillating magnetic field over stretchable rotating disk. Journal of Magnetism and Magnetic Materials, 465: 531-539. http://dx.doi.org/10.1016/j.jmmm.2018.06.019

[25] Zeeshan, A., Maskeen, M.M., Mehmood, O.U. (2018). Hydromagnetic nanofluid flow past a stretching cylinder embedded in non-Darcian Forchheimer porous media. Neural Computing and Applications, 30(11): 3479-3489. http://dx.doi.org/10.1007/s00521-017-2934-7

[26] Khan, S.K., Sanjayanand, E. (2005). Viscoelastic boundary layer flow and heat transfer over an exponential stretching sheet. International Journal of Heat and Mass Transfer, 48(8): 1534-1542. http://dx.doi.org/10.1016/j.ijheatmasstransfer.2004.10.0 32

[27] Zhu, J., Zheng, L., Zhang, Z. (2010). Effects of slip condition on MHD stagnation-point flow over a powerlaw stretching sheet. Applied Mathematics and Mechanics, 31(4): 439-448. http://dx.doi.org/10.1007/s10483-010-0404-z

[28] Mehmood, A. (2017). Viscous Flows: Stretching and Shrinking of Surfaces. Springer. http://dx.doi.org/10.1007/978-3-319-55432-7

[29] Andersson, H.I., Hansen, O.R., Holmedal, B. (1994). Diffusion of a chemically reactive species from a stretching sheet. International Journal of Heat and Mass Transfer, 37(4): 659-664. http://dx.doi.org/10.1016/0017-9310(94)90137-6

[30] Majeed, A., Zeeshan, A., Noori, F. (2019b). Numerical study of Darcy-Forchheimer model with activation energy subject to chemically reactive species and momentum slip of order two. AIP Advances, 9(4): 045035. http://dx.doi.org/10.1063/1.5095546

[31] Prasad, K., Abel, S., Datti, P. (2003). Diffusion of chemically reactive species of a non-Newtonian fluid immersed in a porous medium over a stretching sheet. International Journal of Non-Linear Mechanics, 38(5): 651-657. http://dx.doi.org/10.1016/S00207462(01)00122-6

[32] Shehzad, S., Hayat, T., Qasim, M., Asghar, S. (2013). Effects of mass transfer on MHD flow of Casson fluid with chemical reaction and suction. Brazilian Journal of Chemical Engineering, 30(1): 187-195. http://dx.doi.org/10.1590/S0104-66322013000100020

[33] Bestman, A. (1990). Natural convection boundary layer with suction and mass transfer in a porous medium. International Journal of Energy Research, 14(4): 389-396. http://dx.doi.org/10.1002/er.4440140403

[34] Makinde, O., Olanrewaju, P., Charles, W. (2011). Unsteady convection with chemical reaction and radiative heat transfer past a flat porous plate moving through a binary mixture. Afrika Matematika, 22(1): 6578. http://dx.doi.org/10.1007/s13370-011-0008-z

[35] Maleque, K. (2013). Effects of exothermic/endothermic chemical reactions with Arrhenius activation energy on MHD free convection and mass transfer flow in presence of thermal radiation. Journal of Thermodynamics. http://dx.doi.org/10.1155/2013/692516

[36] Awad, F.G., Motsa, S., Khumalo, M. (2014). Heat and mass transfer in unsteady rotating fluid flow with binary chemical reaction and activation energy. PloS One, 9(9): e107622. http://dx.doi.org/10.1371/journal.pone.0107622

[37] Abbas, Z., Sheikh, M., Motsa, S. (2016). Numerical 
solution of binary chemical reaction on stagnation point flow of Casson fluid over a stretching/shrinking sheet with thermal radiation. Energy, 95: 12-20. http://dx.doi.org/10.1016/j.energy.2015.11.039

[38] Mustafa, M., Khan, J.A., Hayat, T., Alsaedi, A. (2017). Buoyancy effects on the MHD nanofluid flow past a vertical surface with chemical reaction and activation energy. International Journal of Heat and Mass Transfer, 108:

1340-1346. http://dx.doi.org/10.1016/j.ijheatmasstransfer.2017.01.0 29

[39] Ellahi, R., Zeeshan, A., Hussain, F., Asadollahi, A. (2019). Peristaltic blood flow of couple stress fluid suspended with nanoparticles under the influence of chemical reaction and activation energy. Symmetry, 11(2): 276. http://dx.doi.org/10.3390/sym11020276

[40] Dero, S., Uddin, M.J., Rohni, A.M. (2019). Stefan blowing and slip effects on unsteady nanofluid transport past a shrinking sheet: multiple solutions. Heat TransferAsian Research, 48(6): 2047-66. http://dx.doi.org/10.1002/htj.21470

[41] Fang, T., Jing, W. (2014). Flow, heat, and species transfer over a stretching plate considering coupled Stefan blowing effects from species transfer. Communications in Nonlinear Science and Numerical Simulation, $\quad$ 19(9): 3086-97. http://dx.doi.org/10.1016/j.cnsns.2014.02.009

[42] Latiff, N., Uddin, M., Ismail, A.M. (2016). Stefan blowing effect on bioconvective flow of nanofluid over a solid rotating stretchable disk. Propulsion and Power Research, 5(4): 267-78. http://dx.doi.org/10.1016/j.jppr.2016.11.002

[43] Alamri, S.Z., Ellahi, R., Shehzad, N., Zeeshan, A. (2019). Convective radiative plane Poiseuille flow of nanofluid through porous medium with slip: An application of
Stefan blowing. Journal of Molecular Liquids, 273: 292304. http://dx.doi.org/10.1016/j.molliq.2018.10.038

[44] Uddin, M.J., Kabir, M., Bég, O.A. (2016). Computational investigation of Stefan blowing and multiple-slip effects on buoyancy-driven bioconvection nanofluid flow with microorganisms. International Journal of Heat and Mass Transfer, 95: 116-30. http://dx.doi.org/10.1016/j.ijheatmasstransfer.2015.11.0 15

[45] Farooq, U., Zhao, Y., Hayat, T., Alsaedi, A., Liao, S. (2015). Application of the HAM-based Mathematica package BVPh 2.0 on MHD Falkner-Skan flow of nanofluid. Computers \& Fluids, 111: 69-75. http://dx.doi.org/10.1016/j.compfluid.2015.01.005

[46] Liao, S. (2013). Advances in the Homotopy Analysis Method. Singapore, World Scientific, Pages: 428. http://dx.doi.org/10.1142/8939

[47] Zeeshan, A., Shehzad, N., Abbas, T., Ellahi, R. (2019). Effects of radiative electro-magnetohydrodynamics diminishing internal energy of pressure-driven flow of titanium dioxide-water nanofluid due to entropy generation. $\quad$ Entropy, 21(3): 236. http://dx.doi.org/10.3390/e21030236

[48] Misra, J., Shit, G., Chandra, S., Kundu, P. (2011). Hydromagnetic flow and heat transfer of a second-grade viscoelastic fluid in a channel with oscillatory stretching walls: Application to the dynamics of blood flow. Journal of Engineering Mathematics, 69(1): 91-100. http://dx.doi.org/10.1007/s10665-010-9376-x

[49] Zeeshan, A., Shehzad, N., Ellahi, R., Alamri, S.Z. (2018). Convective Poiseuille flow of $\mathrm{Al}_{2} \mathrm{O}_{3}$-EG nanofluid in a porous wavy channel with thermal radiation. Neural Computing and Applications, 30(11): 3371-82. https://doi.org/10.1007/s00521-017-2924-9 